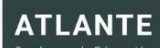

\section{SABERES ANCESTRALES EN LA ENSEÑANZA-APRENDIZAJE EN LA ZONA SUR DE ECUADOR}

\author{
Mónica Hinojosa Becerra \\ Universidad Nacional de Loja \\ monica.hinojosa@unl.edu.ec \\ Ángel Polivio Chalán Chalán² \\ Universidad Nacional de Loja \\ angel.chalan@unl.edu.ec
}

\begin{abstract}
Para citar este artículo puede utilizar el siguiente formato:
Mónica Hinojosa Becerra y Ángel Polivio Chalán Chalán: "Saberes ancestrales en la enseñanzaaprendizaje en la zona sur de Ecuador", Revista Atlante: Cuadernos de Educación y Desarrollo (enero 2021). En línea:

https://www.eumed.net/es/revistas/atlante/2021-enero/saberes-ancestrales-ecuador
\end{abstract}

\begin{abstract}
Resumen
El presente trabajo de investigación tuvo como propósito verificar la aplicación del Modelo del Sistema de Educación Intercultural Bilingüe (MOSEIB) en los Centros Educativos Comunitarios Interculturales Bilingües de la Zona sur de Ecuador. Tiene un enfoque mixto de tipo exploratorio y descriptivo. Para recabar información se aplicó una encuesta construida en base a los criterios e indicadores de evaluación en el proceso de gestión del currículo del MOSEIB, instrumento que fue aplicado al $36 \%$ de los docentes inscritos en Educación Intercultural Bilingüe, pertenecientes al Ministerio de Educación en las Provincias de Loja, Zamora y EI Oro, lo que representa la muestra no probabilística. Se encuestó a 208 de los 570 docentes de educación intercultural. El análisis se apoyó de la teoría y el respaldo legal vigente, llegándose a la conclusión que la aplicación del MOSEIB se da en un $53 \%$ de los casos en un nivel bueno, el $18 \%$ de las ocasiones en un nivel muy bueno, y el restante en niveles regulares y deficientes, ya que existen dificultades que limitan la transferencia de saberes ancestrales. El problema de la pérdida de identidad social es por la limitada formación y capacitación docente, así como también el poco compromiso de la comunidad y todos los miembros del sistema educativo intercultural bilingüe, los cuales deben trabajar mancomunadamente por mantener y rescatar su cultura y su lengua originaria.
\end{abstract}

Palabras claves: Educación Intercultural Bilingüe en Ecuador, Transferencia, Saberes ancestrales de la zona 7 de Ecuador, Modelo del Sistema de Educación Intercultural Bilingüe.

\section{ANCESTRAL KNOWLEDGE IN TEACHING-LEARNING IN THE SOUTH ZONE OF ECUADOR}

\begin{abstract}
The purpose of this research work was to verify the application of the Model of the Bilingual Intercultural Education System (MOSEIB) in the Bilingual Intercultural Community Educational Centers of the southern zone of Ecuador. It has a mixed exploratory and descriptive approach. To collect information, a survey was applied based on the evaluation criteria and indicators in the MOSEIB curriculum management process, an instrument that was applied to $36 \%$ of teachers enrolled in Intercultural Bilingual Education, belonging to the Ministry of Education in the Provinces of Loja, Zamora and El Oro, which represents the non-probabilistic sample. 208 of the 570 intercultural education teachers were surveyed. The analysis was supported by the

${ }^{1}$ Doctora y Licenciada en Comunicación Audiovisual por la Universidad de Málaga. Docente-investigadora de la Universidad Nacional de Loja.

${ }^{2}$ Licenciado en Ciencias de la Educación en la especialización de Lingüística Andina y Educación Bilingüe. Magíster en Estudios de la Cultura. Docente-investigador de la Universidad Nacional de Loja.


theory and current legal support, reaching the conclusion that the application of the MOSEIB occurs in $53 \%$ of the cases at a good level, $18 \%$ of the cases at a very good level, and the remaining at regular and deficient levels, since there are difficulties that limit the transfer of ancestral knowledge. The problem of the loss of social identity is due to the limited training and teacher training, as well as the little commitment of the community and all members of the intercultural bilingual educational system, who must work together to maintain and rescue their culture and language original.

Keywords: Bilingual Intercultural Education in Ecuador, Transfer, Ancestral knowledge of Ecuador's zone 7, Model for the Bilingual and Intercultural Education System.

\section{Introducción}

El desarrollo de la investigación denominada "El proceso educativo en la transferencia de los saberes ancestrales en la Zona sur de Ecuador", que se encuentra normado para su aplicación en los centros educativos comunitarios interculturales bilingües de Ecuador, en el Modelo del Sistema de Educación Intercultural Bilingüe (MOSEIB) (Oviedo \& Wildemeersch, 2008). Se trabajó en coordinación directa con las autoridades zonales de educación. Cabe resaltar el apoyo incondicional de las autoridades para el cumplimiento de los objetivos planteados, en donde se buscó verificar la aplicación del MOSEIB e identificar debilidades operativas y de infraestructura sobre los educandos y la organización comunitaria de los pueblos originarios, para cuidar y mantener su lengua y cultura.

La presente investigación tiene relevancia porque evidencia la importancia de evaluar y garantizar los procesos de calidad y rendición de cuentas en la Educación Intercultural Bilingüe que, mediante una investigación responsable y participativa, permite recopilar información clara y pertinente. Para ello, se aplicó el uso del método científico, con un enfoque mixto, de tipo exploratorio descriptivo, apoyado en al análisis y síntesis para la interpretación de los resultados obtenidos en la aplicación de los instrumentos; y la inducción y deducción que permitió llegar a conclusiones y recomendaciones para solucionar la problemática. Se utilizó la estadística descriptiva para exponer los resultados en las tablas y gráficas.

Se evidenció que el MOSEIB tiene algunas falencias en su aplicación que deben ser modificados aspectos que tienen que ver con la realidad de las infraestructuras, los docentes de los CECIBs (Centros Educativos Comunitarios Interculturales Bilingües) de la Zona 7 y enfocarse en la gestión curricular de la lengua y cultura. Se hace énfasis en las limitaciones que tienen los representantes, docentes, líderes, y todos los actores encargados de la Educación Intercultural Bilingüe para eludir la disminución de identidad social de los pueblos originarios.

\section{Importancia del problema}

La preocupación se basó en la dicotomía existente entre el discurso dictaminado desde el Estado, a través del Ministerio de Educación con relación a la Educación Intercultural Bilingüe, y la verdadera práctica educativa. La Educación Intercultural Bilingüe es un derecho que fue reconocido en la Constitución del 2008. Desde ese momento hasta la actualidad no se evidencia correspondencia entre el reconocimiento constitucional de este derecho, y las prácticas educativas en estas instituciones de atención indígena. Ello se ha visto reflejado en la pérdida de logros, de identidad, autonomía y de derechos educativos luchados y perseguidos a partir del levantamiento de 1990, donde la Confederación de Nacionalidades Indígenas del Ecuador (CONAIE) respaldó este sueño (Almeida, 1991). Ecuador se conforma así con la reproducción de un modelo educativo homogeneizador, que está cada vez más extendido, defendido y perfeccionado por el Estado, y que se sigue legitimando con el lenguaje de la interculturalidad, plurinacionalidad, identidades diversas y la Revolución Ciudadana del Sumak Kawsay (Buen Vivir) (Lara \& de la Herrán Gascón, 2016).

Este proceso, que está teniendo lugar a partir de los discursos de interculturalidad y plurinacionalidad en los archivos constitucionales y jurídicos, no ha quedado más que en eso (archivos); ya que, aparte de lo escrito, no se ha podido ver nada reflejado en la práctica educativa. No se han notado repercusiones reales sobre el sector indígena, ni sobre la sociedad ecuatoriana. Este proceso está conduciendo hacia una relación neocolonial entre gobierno y movimiento indígena, y, por otro lado, en una neocolonización social y étnica sobre las propias comunidades indígenas (Lara Lara, 2019).

En los últimos años el concepto de interculturalidad ha entrado en un debate por la polisemia de su significado. En Latinoamérica, concretamente en Ecuador, la interculturalidad es inherente a las relaciones coloniales entre mestizos, indígenas, criollos y blancos. Dichas relaciones, aun en el siglo XXI, son asimétricas y siguen siendo una herencia histórica y estructural (Dietz \& Mateos, 2001). La interculturalidad no es sólo un concepto sino que constituye una herramienta eficaz para generar procesos sostenidos de decolonialidad (Walsh, 2009) y redefinición de las relaciones establecidas entre los indígenas y el resto de la sociedad ecuatoriana y entre los indígenas y el destinatario de sus demandas, el Estado y los gobiernos de turno.

La educación intercultural, junto con el derecho a la tierra y al territorio, representan la reivindicación del movimiento indígena como camino para resignificar las relaciones interétnicas en Ecuador (Barnach-Calbó, 1997; Dávalos, 2002; Bretón Solo de Saldívar, 2009; Guerrero y Ospina, 2003). No es una apuesta solo por 
el reconocimiento de la pluralidad social que caracteriza a las políticas del multiculturalismo (Koldorf, 2010), sino una vía de escape para la refundación de procesos, para recrear las bases étnicas y culturales que identifican al Estado ecuatoriano, y están acordes con la Constitución del Buen Vivir (Rodríguez Cruz, 2017). La legislación encaminada al reconocimiento de los derechos de los pueblos indígenas no tiene efectos en la vida práctica (Mato, 2009). Existe una gran diferencia entre la teoría de las leyes y normas y la práctica educativa, de manera tal que aunque el Estado ecuatoriano establezca legalmente un sistema de Educación Intercultural Bilingüe, a través de los resultados de esta investigación y el análisis de esta realidad, se evidencia que la escuela y la sociedad siguen resistiéndose al proceso de interculturalización. Uno de esos obstáculos es el anclaje y arraigo de la institución escolar a la pedagogía homogeneizadora y centralista del Estado (Dietz \& Mateos, 2001). La creación de los países latinoamericanos está basado en una estructura interna desigual y heterogénea. En este proceso Ecuador ha sido un país con fracturas sociales más profundas. La anarquía después de la Independencia se alargó en el tiempo frente a otros países del entorno (como Chile o Costa Rica) (Newland, 1991).

\section{Metodología}

Esta investigación presenta un enfoque de carácter mixto, es decir cuali-cuantitativo, de tipo exploratorio descriptivo, utilizando el método científico, analítico y sintético, la deducción y la inducción, y la estadística descriptiva, que permitió las tabulaciones y construcción de tablas y gráficas. Se utilizó como técnica para la recolección de información empírica la encuesta (Marín-Gutiérrez, Andrade-Vargas \& Iriarte Solano, 2016). La misma que se basó en los lineamientos de evaluación propuestos en el MOSEIB, y algunas interrogantes planteadas por la Dirección Zonal de Educación Intercultural Bilingüe. El muestreo se realizó de manera no probabilística, ya que entraron en la muestra quienes respondieron la encuesta vía correo electrónico. El objetivo general fue determinar el nivel de aplicación del Modelo del Sistema de Educación Intercultural Bilingüe (MOSEIB) en los Centros Educativos Comunitarios Interculturales Bilingües de la Zona 7, que considera las provincias de El Oro, Loja y Zamora, dentro de la Política Pública de Educación Intercultural Bilingüe, para fortalecer y preservar la cultura y las tradiciones autóctonas como parte de la riqueza social.

El análisis e interpretación de los resultados se realizó en base a las respuestas otorgadas por 208 docentes, que viene a representar el $36.49 \%$ de la totalidad de docentes. La muestra es de tipo no probabilística, ya que, de acuerdo con los listados registrados en la Dirección de Educación Intercultural Zona 7, la población es de 570 docentes.

El objetivo general de este trabajo fue verificar la aplicación del Modelo del Sistema de Educación Intercultural Bilingüe (MOSEIB) en los Centros Educativos Comunitarios Interculturales Bilingües de Educación General Básica y Bachillerato de la Zona sur de Ecuador, en relación con el currículo.

\section{Resultados}

EI MOSEIB es un modelo educativo práctico que parte de las necesidades e intereses de las nacionalidades y pueblos indígenas de Ecuador. En ella se consideran las formas particulares de vida, se promueve su cultura, contribuyendo al fortalecimiento social de las etnias ecuatorianas. Fomenta la participación activa de los padres de familia, los líderes comunitarios, de los docentes y los estudiantes (Quispe Lema, 2013).

Tabla 1. Promedio total de los resultados encuestas realizadas a 208 docentes Zona 7.

\begin{tabular}{|c|c|c|c|c|c|}
\hline No. & La Educación intercultural contribuye a: & $\begin{array}{l}\text { Muy } \\
\text { Bueno }\end{array}$ & Bueno & Regular & Deficiente \\
\hline 1 & $\begin{array}{l}\text { Atender las necesidades psicológicas } \\
\text { Pedagógicas y socio-culturales de los niños }\end{array}$ & $34 \%$ & $55 \%$ & $11 \%$ & $0 \%$ \\
\hline 2 & $\begin{array}{l}\text { Preparar a los estudiantes en los diferentes } \\
\text { conocimientos y prácticas para la vida }\end{array}$ & $26 \%$ & $54 \%$ & $15 \%$ & $5 \%$ \\
\hline 3 & $\begin{array}{l}\text { Desarrollar actitudes de investigación y } \\
\text { promover la autoeducación en los estudiantes }\end{array}$ & $7 \%$ & $54 \%$ & $38 \%$ & $1 \%$ \\
\hline 4 & Incorporar a la educación, los conocimientos y & $15 \%$ & $58 \%$ & $25 \%$ & $2 \%$ \\
\hline
\end{tabular}


5

Utilizar las lenguas de las nacionalidades como medio de comunicación oral y escrito en todas las áreas del conocimiento

6

Desarrollar el léxico y la expresión oral y escrita de cada una de las lenguas ancestrales

7
$5 \%$

$60 \%$

$32 \%$

$3 \%$

$6 \%$

$49 \%$

$37 \%$

$8 \%$

$36 \%$

$45 \%$

$10 \%$

$9 \%$ kichwa

PROMEDIO

$18 \% \quad 53 \% \quad 24 \%$

$5 \%$

Fuente: Elaboración propia

El cumplimiento del currículum en el proceso de investigación arroja un 53\% en un nivel bueno. Es necesario reconocer que las mayores debilidades se tienen en utilizar las lenguas de las nacionalidades como medio de comunicación oral y escrito en todas las áreas del conocimiento, pues apenas el 18\%, de los encuestados responden que su cumplimiento es muy bueno, ya que manifiestan que la mayoría de los docentes, en general, no utilizan las lenguas de las nacionalidades. Por la utilidad y falta de práctica en el diario vivir de las comunidades se aplica en sus clases el lenguaje castellano. En la gran mayoría de los casos las lenguas originarias solo son una asignatura más. Otra de las debilidades que se pudo detectar es en los resultados que nos arroja la pregunta: Incorporar a la educación los conocimientos y características de cada cultura de Ecuador y de otras culturas del mundo, pues un $24 \%$ lo califica como regular y un $5 \%$ como deficiente.

\section{Discusión}

EI MOSEIB es un modelo elaborado por las nacionalidades y pueblos para el ejercicio pleno de sus derechos colectivos. Es así que los pueblos originarios, por medio de este sistema educativo, luchan por la permanencia de la cultura y las lenguas maternas (Villacís Miranda, 2017). Este trabajo de investigación a través del objetivo propuesto, permitió verificar la aplicación del MOSEIB en los CECIBs de educación en la Zona 7, a través de la encuesta aplicada a los docentes.

Se verificó que el nivel de aplicación de los objetivos del MOSEIB se encuentra en un rango de aplicación bueno con un $53 \%$, un $18 \%$ en un nivel bueno y el $29 \%$ en un nivel entre regular y malo. Lo que permite inferir el cumplimiento medianamente aceptable. Más allá del compromiso de las autoridades, de los docentes, padres de familia, el mantenimiento de los procesos, las limitaciones en infraestructura y recursos, no permiten que el MOSEIB cumpla sus objetivos de forma efectiva (Bayas Vallejo \& Maila Mayla, 2016). Otro aspecto a considerar es que existen diferencias entre los Centros de Educación Intercultural Bilingües de las parroquias urbanas y de las parroquias rurales, en temas de dotación de servicios, tanto en infraestructura como en operatividad para garantizar una educación de calidad.

La práctica y utilización de las lenguas maternas se está extinguiendo por alienación cultural en los contextos familiares, escolares y comunitarios. Negándose así a garantizar la educación como lo determina la Constitución de la República de Ecuador (2008) en su Artículo 26, misma que reconoce a la educación como un derecho que las personas ejercen a largo de su vida, siendo este un deber ineludible e inexcusable del Estado. Constituye un área prioritaria de la política pública y de la inversión estatal, garantía de la igualdad e inclusión social y condición fundamental para el buen vivir, en donde las personas, las familias y la sociedad tienen el derecho y la responsabilidad de participar en el proceso educativo.

\section{Conclusiones}

Luego de analizar los resultados de la presente investigación, se llegó a la conclusión de que los objetivos del MOSEIB en su currículo han alcanzado un nivel de cumplimiento o aplicación buena que se refleja en un $53 \%$ de los casos en los Centros Educativos Comunitarios Interculturales Bilingües (CECIBs) de la Zona 7. Un 29\% se agrupa en una aplicación del MOSEIB en el rango regular y deficiente, ya sea por desconocimiento del modelo, falta de procesos de capacitación y acompañamiento por parte de las autoridades educativas, directores, docentes, alumnos, representantes o padres de familia y de todos los miembros comunitarios para rescatar y mantener la cultura y la lengua indígena de la Zona 7 de Ecuador (Loja, Zamora-Chinchipe y El Oro).

Otros factores visibles que han intervenido para limitar el uso y manejo cotidiano de la lengua kichwa en la mayoría de los centros educativos, en la población juvenil y en la gran mayoría de la población adulta, es el propio sistema educativo manejado e impuesto históricamente desde el Estado nacional, con contenidos ajenos a la realidad socio cultural de las comunidades indígenas; así como el uso de la tecnología y la globalización que representan una barrera para la preservación de la cultura autóctona, ya que aprenden otras ajenas a lo contextual e identitario. 
No se verifica el cumplimiento del Artículo 57, en sus numerales 14 y 21, de la Constitución de la República del Ecuador, en referencia a los Derechos de las comunidades, pueblos y nacionalidades, pues ahí se establece: Desarrollar, fortalecer y potenciar el sistema de educación intercultural bilingüe, con criterios de calidad, desde la estimulación temprana hasta el nivel superior, conforme a la diversidad cultural, para el cuidado y preservación de las identidades en consonancia con sus metodologías de enseñanza y aprendizaje.

Las autoridades Zonales deberán recuperar los ritos y saberes mediante la revitalización de la lengua materna, capacitación continua a todos los miembros de la comunidad educativa, la inserción de los aspectos psicológicos, culturales, académicos, y sociales en el contenido curricular de todos los Centros Educativos Comunitarios Interculturales Bilingües, con el fin de potenciar el cumplimiento de los Objetivos del MOSEIB en la Zona 7.

Los sistemas educativos comunitarios deben incluir en sus contenidos curriculares aspectos de acuerdo a la realidad socio cultural de las comunidades originarias. El Ministerio de Educación, a través de la Dirección Zonal y Distritos correspondientes, debe elaborar materiales didácticos y textos actualizados y contextualizados para lograr fortalecer el proceso de recuperación de las lenguas originarias y que sean distribuidos a todas las parroquias urbanas y rurales de forma oportuna y permanente.

Establecer compromisos y acuerdos con todos los actores involucrados de los Centros Educativos para entrar en un proceso de recuperación del ambiente lingüístico, como una garantía para el uso y manejo de la lengua materna en los centros educativos, en donde las familias y la sociedad tienen el derecho y la responsabilidad de participar en este Modelo Educativo para garantizar la permanencia de la lengua y la cultura originaria en la Zona 7.

La Dirección Zonal 7 y los Distritos de Educación, en colaboración con los centros de educación superior, son los actores principales para promover y trabajar talleres con docentes, padres de familia y miembros de la comunidad, enfocados en hacer que se tome conciencia sobre la importancia que tiene la revitalización de la lengua materna como un medio para el fortalecimiento y preservación de la identidad cultural de los pueblos y comunidades.

\section{BIBLIOGRAFÍA}

Almeida, I. (1991). Indios: Una reflexión sobre el levantamiento indígena de 1990. Quito: Editorial Abya Yala. Barnach-Calbó, E. (1997). La nueva educación indígena en lberoamérica. Revista Iberoamericana de Educación, (13): 13-33. https://bit.ly/31QYkQ9

Bayas Vallejo, A. y Maila Mayla, I.P. (2016). Cuadernia basado en el modelo del sistema de educación intercultural bilingüe Moseib, para la enseñanza de ciencias naturales, en el noveno año de educación básica del colegio técnico intercultural bilingüe El Chaquiñán, período 2015-2016. (Tesis de Grado). Quito: UCE.

Bretón Solo de Saldívar, V. (2009). La deriva identitaria del movimiento indígena en los Andes ecuatorianos o los límites de la etnofagia. Carmen Martínez Novo (compiladora), Repensando los movimientos indígenas. Quito: Ministerio de Cultura del Ecuador: 60-121.

Constitución de la República del Ecuador (2008). Quito: Tribunal Constitucional del Ecuador. Registro oficial Nro, 449. https://bit.ly/3jAnVTw

Dávalos, P. (2002). Movimiento indígena ecuatoriano: Construcción política y epistémica. En Daniel Mato Estudios y otras prácticas intelectuales latinoamericanas en cultura y poder. Caracas: Consejo Latinoamericano de Ciencias Sociales (CLACSO) y CEAP, FACES, Universidad Central de Venezuela: 89-98. https://bit.ly/34wg8lj

Dietz, G. \& Mateos, L. (2001). Interculturalidad y educación intercultural en México. Un análisis de los discursos nacionales e internacionales en su impacto en los modelos educativos mexicanos. México D.F.: Secretaría de Educación Pública y Coordinación General de Educación Intercultural y Bilingüe.

Guerrero, A. \& Ospina, P. (2003). El Poder de la Comunidad. Ajuste estructural y movimiento indígena en los Andes ecuatorianos. Buenos Aires: CLACSO.

Koldorf, A. (2010). Multiculturalismo y diversidad: un debate actual. Rosario: Prohistoria Ediciones.

Lara Lara, F. (2019). Sumak Kawsay y Educación Inclusiva en Ecuador: una propuesta desde el personalismo. Acta Scientiarum. Education, 41, e40274-e40274. DOI: https://doi.org/10.4025/actascieduc.v41i1.40274

Lara, F. L., \& de la Herrán Gascón, A. (2016). Reflexiones sobre la educación del sumak kawsay en Ecuador. Araucaria. Revista Iberoamericana de Filosofía, Política y Humanidades, 18(36), 41-58. https://bit.ly/3molHZ2

Marín-Gutiérrez, I.; Andrade Vargas, L. y Iriarte Solano, M. (2016). Diseño de proyectos de investigacióndesarrollo y propuestas metodológicas. Loja, Universidad Técnica Particular de Loja.

Mato, D. (2009). Instituciones interculturales de Educación Superior en América Latina. Panorama regional, procesos interculturales de construcción institucional, logros, dificultades, innovaciones y desafíos. En Mato, D. (coord.). Instituciones Interculturales de Educación Superior en América Latina. Procesos de construcción, logros, innovaciones y desafíos. Caracas: IESAL-UNESCO. 
Newland, C. (1991). La educación elemental en Hispanoamérica: desde la Independencia hasta la centralización de los sistemas educativos nacionales. Hispanic American Historical Review, (71), 335-364.

Oviedo, A., \& Wildemeersch, D. (2008). Intercultural education and curricular diversification: the case of the Ecuadorian Intercultural Bilingual Education Model (MOSEIB). Compare, 38(4), 455-470. DOI: https://doi.org/10.1080/03057920701860137

Quispe Lema, C. (2013). Educación intercultural y bilingüe. https://bit.ly/325HfIR

Rodríguez Cruz, M. (2017). Interculturalidad, plurinacionalidad y sumak kawsay en Ecuador. La construcción de un nuevo modelo de Estado a través de la educación intercultural bilingüe: discurso y realidad. Perfiles educativos, 39(157), 70-86. https://bit.ly/37J3Yrn

Villacís Miranda, M. P. (2017). El impacto sociocultural que ha marcado la incorporación de la lengua kichwa con el modelo del sistema de educación intercultural bilingue (MOSEIB) en los niños y niñas del subnivel 2 de la Escuela Intercultural Bilingüe Mushuk Pakari. (Tesis de Maestría). Quito: UCE.

Walsh, C. (2009). Interculturalidad, estado, sociedad. Luchas (de) coloniales de nuestra época. Quito: UASB-Abya-Yala. 